\title{
Validación del índice de comorbilidad para trasplante hematopoyético en una cohorte retrospectiva de niños y adolescentes argentinos receptores de trasplante alogénico Validation of the Hematopoietic Cell Transplantation-Specific Comorbidity Index in a retrospective cohort of children and adolescents who received an allogeneic transplantation in Argentina
}

\author{
Dr. Carlos M. Figueroa Turienzo ${ }^{a}$, Dra. Carolina Cernadas ${ }^{b}$, Dra. Mariana Roizen ${ }^{a}$, \\ Dra. Silvia Pizzia y Dra. Raquel Staciuk ${ }^{a}$
}

\section{RESUMEN}

Introducción. El trasplante hematopoyético es una terapia con riesgo de mortalidad relacionada con el trasplante (MRT), que puede variar según la comorbilidad previa. Elíndice de comorbilidad para trasplante hematopoyético (ICTH) es un instrumento desarrollado para medir este riesgo. Los reportes sobre su usoen pediatría son escasos. El objetivo de este estudio fue validar el ICTH en una cohorte pediátrica de receptores de trasplante hematopoyético alogénico en Argentina.

Población y métodos. Cohorte retrospectiva de 140 pacientes trasplantados en el Hospital J. P. Garrahan entre 2008 y 2012. Se revisaron, de las historias clínicas, sus antecedentes y evolución. Se calculó el ICTH de cada paciente y se clasificaron como de riesgo bajo (puntaje 0 ), intermedio (puntaje 1-2) o alto (puntaje $>3$ ). Se estimó la supervivencia para cada grupo por el método de Kaplan-Meier y se comparó con la prueba de logaritmos de rangos. En el caso de las enfermedades malignas, la recaída fue considerada un evento competitivo con la MRT. Se consideró significativa una $\mathrm{p}<0,05$.

Resultados. La mediana del ICTH fue 1 (r: 0-6). El $45,7 \%$ de los pacientes tuvieron puntaje 0 ; el $40,7 \%, 1-2$; y el $13,6 \%$, > 3. Las comorbilidades más frecuentes fueron obesidad, infección y compromiso pulmonar y hepático. La MRT de los pacientes con puntaje 0 fue $14,1 \%$; con puntaje $1-2,43,7 \%$; y con puntaje $>3,52,6 \%$. Las curvas de supervivencia manifestaron diferencias entre los tres grupos ( $\mathrm{p} 0,01)$.

Conclusión. ElICTH mostró ser una herramienta efectiva para predecir el riesgo de MRT en nuestro medio.

Palabras clave: comorbilidad, trasplante de células madre hematopoyéticas, mortalidad no asociada a recaída, pediatría.

http:/ /dx.doi.org/10.5546/aap.2016.337

Financiamiento:

Ninguno.

Conflicto de intereses: Ninguno que declarar.

Recibido: 13-12-2015 Aceptado: 10-2-2016

\section{INTRODUCCIÓN}

A pesar de los avances en el tratamiento de soporte, el trasplante hematopoyético (TH) es aún una terapia con alto riesgo de mortalidad. ${ }^{1}$

El compromiso previo de diferentes órganos o los antecedentes infecciosos pueden aumentar la probabilidad de complicaciones o de mortalidad relacionada con el trasplante (MRT). ${ }^{2-5}$ Cuando, en un paciente, coexisten distintos factores de riesgo, resulta difícil estimar su riesgo individual. Disminuir la incertidumbre al respecto puede ayudar a definir la elegibilidad de un paciente para el TH, la elección del régimen de acondicionamiento más apropiado, y facilitar un adecuado proceso de consentimiento.

Sorror y cols., publicaron, en 2005, los resultados de la validación de un nuevo instrumento útil para acercarse a la mencionada incertidumbre, el índice de comorbilidad para trasplante hematopoyético (ICTH), ${ }^{6}$ diseñado a partir del índice de comorbilidad general de Charlson. ${ }^{7}$ El ICTH les permitió a los autores definir tres categorías de riesgo de MRT según las comorbilidades existentes antes del trasplante. Varios estudios posteriores, que incluyeron dos estudios multicéntricos prospectivos, corroboraron la utilidad de ICTH, ${ }^{8-12}$ aunque hubo también reportes de algunos centros con resultados disímiles. ${ }^{13,14}$

En 2011, Smith y colaboradores describieron los resultados de la aplicación del ICTH a una cohorte retrospectiva de 252 pacientes pediátricos seguidos en 4 centros 
de EE. UU. ${ }^{15}$ El ICTH mostró, en este estudio, un buen poder predictivo de la MRT y la supervivencia global.

El objetivo principal del presente estudio fue evaluar la eficacia del ICTH para predecir el riesgo de MRT y la supervivencia en una cohorte retrospectiva de niños y adolescentes receptores de $\mathrm{TH}$ alogénico en un centro de Argentina. Como objetivos secundarios, se exploraron las causas de MRT y el impacto de la infección fúngica invasiva (IFI) pre- y postrasplante.

\section{POBLACIÓN Y MÉTODOS}

Cohorte restrospectiva compuesta por una muestra consecutiva de 140 pacientes que recibieron un $\mathrm{TH}$ alogénico entre el $1^{\circ}$ de enero de 2008 y el 31 de diciembre de 2012 en el Servicio de Trasplante de Médula Ósea del Hospital de Pediatría Prof. Dr. Juan P. Garrahan.

Todos los pacientes recibieron profilaxis antibiótica con ciprofloxacina y fluconazol durante el período de neutropenia, aciclovir para la profilaxis de herpes simple y trimetoprima y sulfametoxazol o pentamidina para la profilaxis de Pneumocystis jiroveci. El manejo de la infección por citomegalovirus consistió en su tratamiento temprano, basado en la vigilancia semanal de la carga viral. Se administró heparina en bajas dosis para la profilaxis de la enfermedad venooclusiva hepática. La profilaxis de la enfermedad de injerto contra huésped (EICH) consistió en un inhibidor de calcineurina (ciclosporina A en los trasplantes familiares y tacrolimus en los no familiares) asociado a Metotrexato en los receptores de médula ósea o sangre periférica. En los receptores de sangre de cordón umbilical, la profilaxis de EICH consistió en la asociación de un inhibidor de calcineurina con metilprednisona o micofenolato.

La evaluación pretrasplante incluyó estudios de laboratorio con la evaluación de la función renal y hepática, ecocardiograma, ecografía abdominal, tomografía computada de tórax y senos paranasales, y la realización de estudios funcionales respiratorios a partir de los seis años.

Estrategia: Uno de los investigadores revisó la historia clínica de cada paciente, que incluyó una hoja de registro de la evaluación al momento del ingreso a la Unidad de Trasplante. Para el registró de la información concerniente al ICTH, se siguieron las recomendaciones de Sorror. ${ }^{16}$ Cuando fue necesario, se revisó también la base de datos de laboratorio. En caso de duda para la asignación de puntajes, se discutió con el resto de los investigadores.
Se registraron los siguientes datos: sexo, edad al momento del trasplante, diagnóstico, fecha del $\mathrm{TH}$, fecha de muerte relacionada con el trasplante, fecha de recaída, fecha del último control, tipo de donante, tipo de acondicionamiento y fuente de células progenitoras hematopoyéticas. Se registró también el antecedente de IFI previa al TH y los siguientes eventos postrasplante: EICH aguda (grado 2-4 y grado 3-4), EICH crónica (limitada y extensa) e IFI, factores de riesgo reconocidos de MRT. ${ }^{1,5}$

ICTH: El puntaje del índice surge de evaluar 17 ítems al momento de ingresar el paciente para el TH, y le corresponde a cada resultado un puntaje determinado, que surge de su peso como factor de riesgo en el trabajo original. ${ }^{6}$ Se registraron los resultados para cada uno de estos ítems de acuerdo con las especificaciones de los autores y se calculó el puntaje del índice para cada paciente. Se consideraron las mismas categorías de riesgo que en el trabajo original: leve para los pacientes con puntaje 0 , intermedio para los pacientes con puntaje 1-2 y alto para aquellos con puntaje $\geq 3$.

Análisis estadístico: Se resumieron los datos de las distintas variables con porcentajes o mediana y rango, según correspondiera. Se comparó la frecuencia de EICH aguda y crónica, de IFI y de MRT entre los TH familiares y no familiares con la prueba de Fisher. Se analizó, por regresión logística, la asociación entre MRT y los siguientes factores de riesgo: EICH aguda grado 2-4 y grado 3-4, EICH crónica extensa e IFI pre- y postrasplante. Para evaluar la asociación entre la categoría de riesgo del ICTH y el riesgo de MRT, se estimó la supervivencia para cada grupo por el método de Kaplan-Meier y se la comparó con la prueba de logaritmos de rangos (logrank test). En el caso de las enfermedades malignas, la recaída fue considerada un evento competitivo con la MRT. Se consideró significativa una $\mathrm{p}<0,05$. El paquete estadístico usado fue Stata 9.

El protocolo fue evaluado y aprobado por los Comités Revisores de Investigación y de Ética del Hospital.

\section{RESULTADOS}

En la Tabla 1, se describen las características generales de la muestra. Como puede observarse, la principal indicación para el $\mathrm{TH}$ fueron las leucemias agudas, de las cuales el $45,8 \%$ estaba en segunda remisión y el 2,7\%, en tercera.

El 55\% de los pacientes presentó EICH aguda grado $2-4$ y el $11,4 \%$, grado $3-4(8,3 \%$ en los $\mathrm{TH}$ 
familiares vs. 18,3\% en los no familiares, p 0,14).

La frecuencia de EICH crónica extensa fue de $28,6 \%$ ( $25 \%$ en los TH familiares vs. $36,4 \%$ en los no familiares, $\mathrm{p} 0,23)$.

El 7,8\% de los pacientes tenía el antecedente de IFI previa al TH y el 19,3\% presentó IFI luego del trasplante $(15,3 \%$ en los familiares vs. $27,3 \%$ en los no familiares, $\mathrm{p} 0,11$ ).

La MRT fue del 27,1\%, con peor evolución en el caso de los TH no familiares $(19,8 \%$ en los familiares vs. $42,8 \%$ en los no familiares, p 0,007).

Las causas de MRT más frecuentes fueron las infecciones (92\%), asociadas a EICH activa o refractaria a corticoides en el 39\% de los casos. Esta última condición se observó en el 21\% de los casos de EICH.

En la Tabla 2, se describe la frecuencia de infecciones documentadas que fueron causa de MRT.

Se asociaron con una mayor MRT la presencia de EICH aguda grado 3-4 (OR 7,8; IC: 2,4-25,1; p 0,001) y de IFI luego del TH (OR 2,7; IC: 1,1-7; p

TABLA 1. Características generales de los pacientes

\begin{tabular}{ll}
\hline & $\mathbf{n}(\%)$ \\
\hline Total de pacientes & 140 \\
Edad mediana (rango) & 9 años $(0,2-18)$ \\
Varones & $87(62)$ \\
Diagnóstico: & \\
$\quad$ Leucemia aguda & $75(53,6)$ \\
$\quad$ Síndrome mielodisplásico & $15(10,7)$ \\
Anemia aplásica & $14(10)$ \\
Wiskott-Aldrich & $4(2,9)$ \\
$\quad$ Mucopolisacaridosis tipo I & $4(2,9)$ \\
Anemia de Fanconi & $4(2,9)$ \\
Inmunodeficiencia combinada grave & $3(2,1)$ \\
Otras inmunodeficiencias graves & $5(3,6)$ \\
Leucemia mielomonocítica juvenil & $3(2,1)$ \\
Leucemia mieloide crónica & $2(1,4)$ \\
Linfoma & $2(1,4)$ \\
Linfohistiocitosis hemofagocítica & $2(1,4)$ \\
Adrenoleucodistrofia & $2(1,4)$ \\
Eritroblastopenia & $2(1,4)$ \\
Otras* & $3(2,1)$ \\
Tipo de donante: & \\
Familiar idéntico & $96(68,6)$ \\
No familiar idéntico & $44(31,4)$ \\
Fuente hematopoyética: & \\
Médula ósea & $88(62,9)$ \\
Sangre periférica & $29(20,7)$ \\
Sangre de cordón umbilical & $23(16,4)$ \\
Acondicionamiento: & \\
Mieloablativo & $124(88,6)$ \\
No mieloablativo & $16(11,4)$ \\
\hline
\end{tabular}

* Talasemia, síndrome de Shwachman-Diamond, hemoglobinuria paroxística nocturna.
0,039). No se demostró asociación entre la MRT y el antecedente de IFI (OR 1,4; IC: 0,4-5,9) o la presencia de EICH crónica extensa (OR 1,1; IC: $0,4-2,3)$.

\section{Índice de comorbilidad para trasplante hematopoyético}

La mediana del puntaje fue de 1 (r: 0-6). El $45,7 \%$ de los pacientes tuvieron un puntaje 0 ; el $40,7 \%, 1-2$; y el $13,6 \%, \geq 3$. No se observaron diferencias significativas entre los receptores de TH familiares y no familiares.

En la Tabla 3, se describen las comorbilidades presentes en la muestra. Las más frecuentes fueron obesidad, infección, compromiso pulmonar y hepático.

TABLA 2. Infecciones documentadas como causa de mortalidad relacionada con el trasplante hematopoyético

\begin{tabular}{ll}
\hline Infecciones & $\mathbf{n}$ \\
\hline Virus & 15 \\
Citomegalovirus & 8 \\
Adenovirus & 5 \\
Virus respiratorios de la comunidad & 2 \\
Bacterias & 6 \\
Bacilos Gram negativos & 5 \\
Mycoplasma pneumoniae & 1 \\
Infecciones fúngicas invasivas & 4 \\
Aspergillus & 3 \\
Candida glabrata & 1 \\
Pneumocystis jiroveci & 2 \\
Toxoplasmosis & 1 \\
\hline
\end{tabular}

TABLA 3. Frecuencia de comorbilidades evaluadas con el indice de comorbilidad para trasplante hematopoyético

\begin{tabular}{ll}
\hline Comorbilidad & $\mathbf{n}(\mathbf{\%})$ \\
\hline Arritmia cardíaca & $1(0,71)$ \\
Valvulopatía & 0 \\
Otras enfermedades cardíacas & 0 \\
Úlcera péptica & 0 \\
Tumor sólido previo & 0 \\
Trastornos psiquiátricos & $5(3,57)$ \\
Obesidad & $28(20)$ \\
Infección & $25(17,86)$ \\
Enfermedad renal moderada/grave & 0 \\
Enfermedades reumáticas & 0 \\
Diabetes & 0 \\
Compromiso pulmonar moderado & $7(5)$ \\
Compromiso pulmonar grave & $19(13,57)$ \\
Compromiso hepático moderado/grave & $8(5,71)$ \\
Compromiso hepático leve & $13(9,9)$ \\
Enfermedad cerebrovascular & $4(2,86)$ \\
Enfermedad inflamatoria intestinal & $1(0,71)$ \\
\hline
\end{tabular}


La MRT de los pacientes con puntaje 0 fue $14,1 \%(10,6 \%$ en familiares vs. $23,5 \%$ en no familiares, $\mathrm{p} 0,23)$, de los pacientes con puntaje $1-2,43,7 \%$ (27,3\% en familiares vs. $80 \%$ en no familiares, $p 0,1)$ y de aquellos con puntaje $\geq$ $3,52,6 \%$ (50\% en familiares vs. $57,1 \%$ en no familiares).

Un puntaje de 0 se asoció con un menor riesgo de MRT tanto en los receptores de $\mathrm{TH}$ familiar (OR 0,30; IC: 0,09-0,9; p 0,03) como en los receptores de TH no familiar (OR 0,25; IC: 0,06$0,95 ; \mathrm{p} 0,04)$.

En los pacientes con comorbilidad (puntaje $>0$ ), se observó una tendencia a presentar mayor frecuencia de IFI postrasplante con respecto a aquellos que no la presentaban ( $25 \%$ vs. $12,5 \%$, p 0,08) y de EICH aguda grado $3-4(15,79$ vs. 6,25\%, p 0,1).

En las Figura 1, pueden observarse las curvas de supervivencia, que muestran diferencias significativas entre los grupos (p 0,01).

\section{DISCUSIÓN}

Los resultados del presente estudio muestran que el ICTH predice el riesgo de MRT de los pacientes pediátricos atendidos en nuestro medio.

La posibilidad de utilizar donantes no familiares, los avances en las técnicas de soporte y la diversificación de los regímenes de acondicionamiento han permitido ampliar la base de pacientes candidatos al TH. ${ }^{17}$ Esto lleva a una mayor heterogeneidad de los candidatos a este tratamiento y a la inclusión de pacientes con mayor compromiso orgánico previo al trasplante, por lo que se torna más difícil tanto la comparación de distintos grupos como la predicción individual de la chance de supervivencia y del riesgo de MRT.

El índice de Charlson, utilizado para medir comorbilidad en distintas enfermedades, mostró, combinado con el estado funcional, un buen poder predictivo de MRT, pero limitaciones en su sensibilidad para captar comorbilidades en los candidatos a TH. ${ }^{18}$ Sorror y colaboradores desarrollaron, a partir de él, un nuevo indicador, el ICTH, que, con la incorporación de comorbilidades frecuentes en los receptores de $\mathrm{TH}$, como son las infecciones y los trastornos psiquiátricos, demostró una mayor sensibilidad y poder predictivo. ${ }^{6}$

Aunque la muestra con la que se construyó y validó el ICTH incluyó pacientes pediátricos (los autores describieron un rango de edades de 0,8 a 72,7 años), la mediana de edad fue 44 años y los niños y adolescentes no fueron analizados como subgrupo. Smith y cols., publicaron, en 2011, el único estudio que hemos hallado que utilice el ICTH en una población exclusiva de niños y adolescentes. ${ }^{15}$ Con variaciones entre los distintos centros participantes, los autores describieron que el $55 \%$ de sus pacientes tenían un puntaje de 0 ; un $21 \%$, de $1-2$; y un $24 \%$, de 3 o más. El porcentaje de pacientes sin comorbilidad en esa muestra fue superior al hallado en el estudio de validación original del ICTH, en el que el porcentaje de pacientes con puntaje 0 fue $38 \%$ y otro $38 \%$ tuvo un puntaje igual o mayor a $3 .^{6}$

En nuestra muestra, el porcentaje de pacientes con puntaje $0,46 \%$, ocupa un valor intermedio, pero el porcentaje de pacientes con riesgo alto fue menor al de los estudios mencionados.

Una pregunta que surge de estos datos es si los receptores pediátricos de $\mathrm{TH}$ tienen menos comorbilidades que los pacientes de mayor

FIGURA 1. Curvas de supervivencia por grupos de riesgo según el índice de comorbilidad para el trasplante de células progenitoras hematopoyéticas

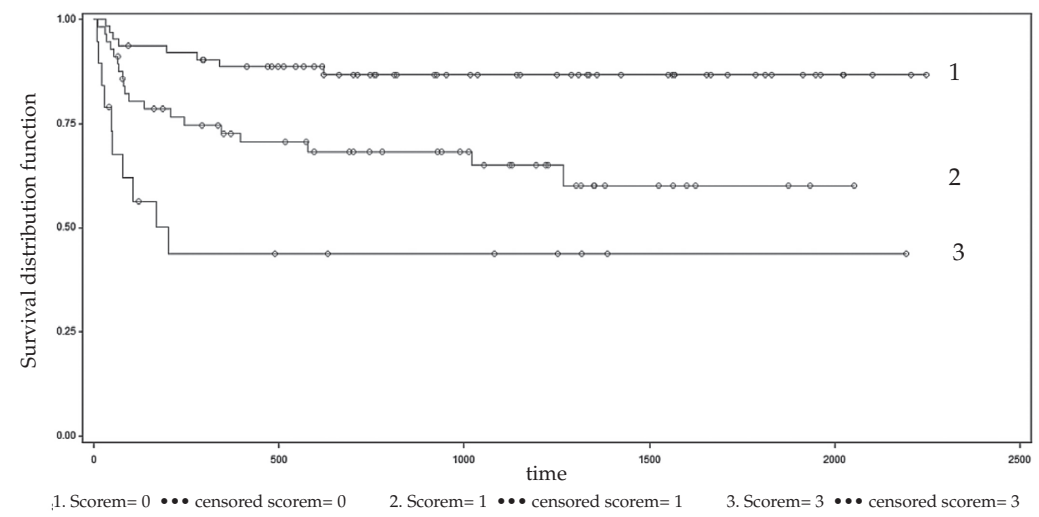


edad y/o si tienen comorbilidades específicas que el ICTH no capta. Aunque la prevalencia de comorbilidades aumenta con la edad, ${ }^{19}$ algunos factores podrían afectar la sensibilidad del ICTH en pediatría. En primer lugar, algunas enfermedades pediátricas con indicación de $\mathrm{TH}$, como los errores congénitos del metabolismo o las fallas medulares constitucionales, tienen comorbilidades asociadas, como malformaciones o trastornos del desarrollo que el ICTH no registra. En segundo lugar, el límite de creatininemia de $2 \mathrm{mg} \%$ (valor que, en nuestro centro, es considerado como criterio para no ser elegible para trasplante) para considerar compromiso renal grave puede subvalorar caídas significativas del filtrado glomerular en niños pequeños con valores de creatinina más bajos. La estimación del filtrado glomerular al agregar a la creatininemia factores de corrección que consideran la edad y/o datos antropométricos es una práctica corriente en pediatría que merecería ser evaluada en futuros estudios. ${ }^{20}$ Las dificultades para realizar estudios funcionales respiratorios en pacientes menores que la edad escolar pueden también ser otra causa de subregistro de compromiso respiratorio en ese grupo etario. La incorporación de las nuevas técnicas de estudio funcional respiratorio en lactantes y preescolares, cada vez más utilizadas en el seguimiento de aquellos pacientes con antecedentes clínicos significativos en la práctica asistencial, podría mejorar este aspecto de la evaluación. ${ }^{21}$

El compromiso pulmonar y hepático, junto con las infecciones, fueron las comorbilidades más frecuentes halladas en la cohorte pediátrica estudiada por Smith y cols..$^{15}$ En nuestra cohorte, estas comorbilidades siguieron en frecuencia a la obesidad que, con una prevalencia de $20 \%$, fue la más habitual. Este porcentaje es mucho mayor que el referido por Smith y cols., en cuya muestra, la obesidad apenas supera el 1,1\%, pero esta diferencia puede deberse a cuestiones metodológicas. Mientras que, en el trabajo citado, los autores tomaron como criterio de obesidad un índice de masa corporal igual o superior a $35 \mathrm{~kg} / \mathrm{m}^{2}$, criterio utilizado en adultos, nosotros consideramos como límite el percentil 95 para la edad siguiendo las recomendaciones publicadas posteriormente por Sorror. ${ }^{16}$ De acuerdo con este criterio, la prevalencia de obesidad, en nuestra muestra, es aún inferior a la encontrada por otros autores, como White y cols..$^{22}$

Las infecciones asociadas o no a la EICH fueron la principal causa de MRT en nuestra muestra. Esto coincide con estudios previos sobre la MRT. ${ }^{23}$ La literatura aporta datos que ayudan a entender la relación entre la comorbilidad previa al TH y estos eventos. Sorror y cols., reportaron, en un estudio multicéntrico reciente, una asociación entre el ICTH y el riesgo de formas más graves de $\mathrm{EICH},{ }^{24}$ lo que coincide con la tendencia observada en nuestra muestra. Además, Bayraktar y cols., hallaron que el ICHT se asociaba con una mayor MRT en los receptores de TH que ingresaban a Cuidados Intensivos. ${ }^{25}$ Siendo la EICH grave un factor de riesgo de infecciones, ${ }^{26}$ resulta lógico pensar que la asociación más frecuente entre comorbilidad y MRT en pacientes pediátricos es consecuencia, en la mayoría de los casos, de la falla de órganos secundaria a la interacción entre la EICH y las infecciones que la complican, más que de la falla primaria de órganos por efecto del acondicionamiento.

$\mathrm{Al}$ analizar la frecuencia de MRT en nuestra muestra, se ve una diferencia significativa entre los receptores de $\mathrm{TH}$ familiares y no familiares. Aunque estudios realizados en algunos centros con mucha experiencia en trasplante no familiar reportan supervivencias similares con ambos tipos de donantes, ${ }^{27}$ otros sí muestran diferencias en la MRT según el tipo de donante, diferencia que ha tendido a disminuir en los últimos años. ${ }^{28}$ Durante el período estudiado, la mediana de tiempo en nuestro centro desde el inicio de la búsqueda de un donante no familiar hasta la realización del trasplante fue de 6 meses. Esta demora expone a los pacientes con enfermedades malignas a recibir mayores dosis acumuladas de quimioterapia y a aquellos con falla hematológica o inmunodeficiencias a un mayor riesgo de infecciones o sobrecarga de hierro. Sin embargo, en nuestro estudio, no hemos observado que esta demora se reflejara en una puntuación mayor del ICTH en los receptores de trasplantes no familiares. Esto podría deberse al reducido número de receptores de $\mathrm{TH}$ no familiar en nuestra muestra o podría señalar limitaciones en la sensibilidad del ICTH.

Al respecto, resulta de interés un trabajo de Vaughn y cols., de reciente publicación, en el que muestran que la combinación del ICTH con marcadores biológicos, como la ferritina, la albúmina y el recuento plaquetario, aumenta el poder discriminativo del instrumento. ${ }^{29}$

La conclusión de nuestro trabajo es que el ICTH es una herramienta útil para la predicción de supervivencia y la evaluación del riesgo de mortalidad en los niños y adolescentes receptores 
de TH en nuestro medio. Su aplicación en la asistencia e investigación puede contribuir a un mejor proceso de consentimiento y a la toma de decisiones en la planificación y gestión del trasplante. $^{30}$

\section{REFERENCIAS}

1. Gooley TA, Chien JW, Pergam SA, Hingorani S, et al. Reduced mortality after allogeneic hematopoietic-cell transplantation. N Engl J Med 2010;363(22):2091-101.

2. Mancuzo EV, Rezende NA. Hematopoietic stem cell transplantation: pulmonary function tests and posttransplant mortality. J Bras Pneumol 2011;37(5):598-606.

3. Hertenstein B, Stefanic M, Schmeiser T, Scholz M, et al. Cardiac toxicity of bone marrow transplantation: predictive value of cardiologic evaluation before transplant. J Clin Oncol 1994;12(5):998-1004.

4. Lee SH, Yoo KH, Sung KW, Koo HH, et al. Hepatic venoocclusive disease in children after hematopoietic stem cell transplantation: incidence, risk factors, and outcome. Bone Marrow Transplant 2010;45(8):1287-93.

5. Martino R, Parody R, Fukuda T, Maertens J, et al. Impact of theintensity of the pretransplantation conditioning regimen in patients with prior invasive aspergillosis undergoing allogeneic hematopoietic stem cell transplantation: a retrospective survey of the Infectious Diseases Working Party of the European Group for Blood and Marrow Transplantation. Blood 2006;108(9):2928-36.

6. Sorror ML, Maris MB, Storb R, Baron F, etal. Hematopoietic cell transplantation (HCT)-specific comorbidity index: a new tool for risk assessment before allogeneic HCT. Blood 2005;106(8):2912-9.

7. Charlson ME, Pompei P, Ales KL, MacKenzie CR. A new method of classifying prognostic comorbidity in longitudinal studies: development and validation.JChronic Dis 1987;40(5):373-83.

8. Raimondi R, Tosetto A, Oneto R, Cavazzina R, et al. Validation of the Hematopoietic Cell TransplantationSpecific Comorbidity Index: a prospective, multicenter GITMO study. Blood 2012;120(6):1327-33.

9. Sperr WR, Wimazal F, Kundi M, Baumgartner C, et al. Comorbidity as prognostic variable in MDS: comparative evaluation of the HCT-CI and CCI in a core dataset of 419 patients of the Austrian MDS Study Group. Ann Oncol 2010;21(1):114-9.

10. Sorror ML, Giralt S, Sandmaier BM, De Lima M, et al. Hematopoietic cell transplantation specific comorbidity index as an outcome predictor for patients with acute myeloid leukemia in first remission: combined FHCRC and MDACC experiences. Blood 2007;110(13):4606-13.

11. Zipperer E, Pelz D, Nachtkamp K, Kuendgen A, et al. The hematopoietic stem cell transplantation comorbidity index is of prognostic relevance for patients with myelodysplastic syndrome. Haematologica 2009;94(5):729-32.

12. Sorror ML, Logan BR, Zhu X, Rizzo JD, et al. Prospective validation of the predictive power of the hematopoietic cell transplantation comorbidity index: a center for International Blood and Marrow Transplant Research Study. Biol Blood Marrow Transplant 2015;21(8):1479-87.

13. Guilfoyle R, Demers A, Bredeson C, Richardson E, et al. Performance status, but not the hematopoietic cell transplantation comorbidity index (HCT-CI), predicts mortality at a Canadian transplant center. Bone Marrow Transplant 2009;43(2):133-9.

14. Birninger N, Bornhäuser M, Schaich M, Ehninger G, et al. Thehematopoietic cell transplantation-specific comorbidity index fails to predict outcomes in high-risk AML patients undergoing allogeneic transplantation- investigation of potential limitations of the index. Biol Blood Marrow Transplant 2011;17(12):1822-32.
15. Smith AR, Majhail NS, MacMillan ML, DeFor TE, et al. Hematopoietic cell transplantation comorbidity index predicts transplantation outcomes in pediatric patients. Blood 2011;117(9):2728-34.

16. Sorror ML. How Iassess comorbidities before hematopoietic cell transplantation. Blood 2013;121(15):2854-63.

17. Storb RF, Champlin R, Riddell SR, Murata M, et al. Non-myeloablative transplants for malignant disease. Hematology Am Soc Hematol Educ Program 2001:375-91.

18. Artz AS, Pollyea DA, Kocherginsky M, Stock W, et al. Performance status and comorbidity predict transplantrelated mortality after allogeneic hematopoietic cell transplantation. Biol Blood Marrow Transplant 2006;12(9): 954-64.

19. Broemeling AM, Watson DE, Prebtani F. Population patterns of chronic health conditions, co-morbidity and healthcare use in Canada: implications for policy and practice. Healthc Q 2008;11(3):70-6.

20. Blufpand HN, Westland R, van Wijk JA, Roelandse-Koop EA, et al. Height-independent estimation of glomerular filtration rate in children: an alternative to the Schwartz equation. J Pediatr 2013;163(6):1722-7.

21. Lum S. Lung function in preschool children: applications in clinical and epidemiological research. Paediatr Respir Rev 2006;7(Suppl 1):S30-2.

22. White M, Murphy AJ, Hallahan A, Ware RS, et al. Survival in overweight and underweight children undergoing hematopoietic stem cell transplantation. Eur J Clin Nutr 2012;66(10):1120-3.

23. Mateos MK, O'Brien TA, Oswald C, Gabriel M, et al. Transplant-related mortality following allogeneic hematopoeitic stem cell transplantation for pediatric acute lymphoblastic leukemia: 25-year retrospective review. Pediatr Blood Cancer 2013;60(9):1520-7.

24. Sorror ML, Martin PJ, Storb RF, Bhatia S, et al. Pretransplant comorbidities predict severity of acute graft-versus-host disease and subsequent mortality. Blood 2014;124(2): 287-95.

25. Bayraktar UD, Shpall EJ, Liu P, Ciurea SO, et al. Hematopoietic cell transplantation-specific comorbidity index predicts inpatient mortality and survival in patients who received allogeneic transplantation admitted to the intensive care unit. J Clin Oncol 2013;31(33):4207-14.

26. Castagnola E, Bagnasco F, Bandettini R, Caviglia I, etal. Role of acute graft-versus-host disease in the risk of bacteremia and invasive fungal disease after allogeneic hemopoietic stem cell transplantation in children. Results from a singlecenter observational study. Biol Blood Marrow Transplant 2014;20(7):1068-73.

27. Leung W, Campana D, Yang J, Pei D, et al. High success rate of hematopoietic cell transplantation regardless of donor source in children with very high-risk leukemia. Blood 2011;118(2):223-30.

28. Moore AS, Shaw PJ, Hallahan AR, Carter TL, et al. Haemopoietic stem cell transplantation for children in Australia and New Zealand, 1998-2006: a report on behalf of the Australasian Bone Marrow Transplant Recipient Registry and the Australian and New Zealand Children's Haematology Oncology Group. Med J Aust 2009;190(3): 121-5.

29. Vaughn JE, Storer BE, Armand P, Raimondi R, et al. Design and validation of an augmented hematopoietic cell transplantation-comorbidity index comprising pretransplant ferritin, albumin, and platelet count for prediction of outcomes after allogeneic transplantation. Biol Blood Marrow Transplant 2015;21(8):1418-24.

30. Bejanyan N, Bolwell BJ, Lazaryan A, Rybicki L, et al. Risk factors for 30-day hospital readmission following myeloablative allogeneichematopoietic cell transplantation (allo-HCT). Biol Blood Marrow Transplant 2012;18(6):874-80. 